\title{
Proposal for a temporal version of a hypertelescope
}

\author{
F. Reynaud and L. Delage \\ Équipe Photonique - Xlim (CNRS UMR 6172), University of Limoges, 123 avenue Albert Thomas, 87060 Limoges Cedex, France \\ e-mail: francois.reynaud@unilim.fr
}

Received 23 May 2006 / Accepted 6 December 2006

\begin{abstract}
Aims. We propose a new version of hypertelescope devoted to high-resolution direct imaging.

Methods. By using a temporal versus spatial optical-path modulation in a multi-aperture interferometer, we show that the beam densification process can be similarly achieved in the time domain giving rise to a good reconfiguration versatility. Reducing the span of optical modulations allows us to convert this instrument into a nulling interferometer.

Results. This new experimental configuration allows an easy and versatile recombination reconfiguration for a ground or space instrument and answers the problem of the variable spatial Fourier spectrum sampling either with a ground-based observation or for a pupil reconfiguration for a space mission.
\end{abstract}

Key words. techniques: high angular resolution - techniques: interferometric - techniques: image processing

\section{Introduction}

This paper reports on the transposition into the temporal domain of the spatial densification in a diluted synthesized aperture by means of the hypertelescope technique. The hypertelescope proposals of Labeyrie (1996) and Vakili et al. (2004) simultaneously use a homothetic reconfiguration according to the "golden rule of imaging interferometers" (Traub 1986) and a densification of the instrument output in the pupil and image planes, respectively. In this way, an angular magnification and a concentration of the energy around the zero order of the interfering beams allow us to optimize the detection properties in a field where, for instance, an exoplanet is expected. The performances of a temporal versus spatial telescope have to be compared conclusively in terms of homothetic reconfiguration and beam densification. The versatility of the time-domain solution is discussed in order to propose an attractive development of the classical hypertelescope concept. In the first part, thanks to the spatial approach of Pedretti et al. (2000) and Gillet et al. (2003), we propose the temporal version of this instrument. In the second part we focus on the advantages of a time-modulated version of this imaging instrument. A versatile reconfiguration and a very high densification are the two main improvements. At last, we show how to convert the imaging instrument into a nulling interferometer using a higher densification potential in the time domain.

\section{Spatial hypertelescopes}

This paragraph treats the proposal of Labeyrie, but note that similar techniques as IRAN (Vakili et al. 2004 and Aristidi et al. 2004) using a beam recombination in the pupil plane are also within the scope of this study. The analytical description is quite different, but the global results and properties can be derived similarly.

Figure 1 recalls the structure of a hypertelescope as proposed by Labeyrie. This simplified drawing does not show the reconfiguration and densification process in detail but shows the main idea. This technique makes it necessary to remap the input

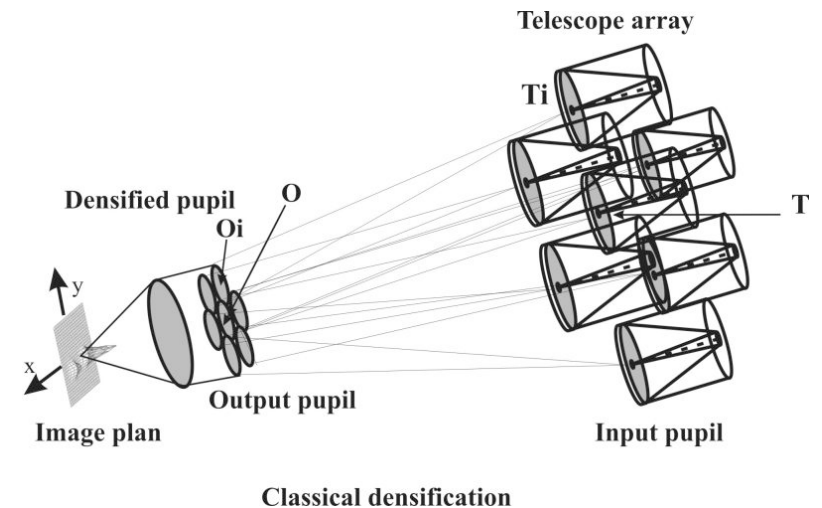

Fig. 1. Spatial hypertelescope: the input pupil is reconfigured with a $G$ contraction ratio and a densification of the output pupil $\left(T T_{i}=G\right.$. $\left.O O_{i}\right)$. The image is displayed as function of spatial coordinates $(x ; y)$ in the image plane.

pupil, making certain to achieve this geometrical contraction using a homothetic process for locating beams centres in the output pupil according to the "golden rule of imaging interferometry". Denoting $G$ as the reconfiguration ratio, the distance between telescope $T_{i}$, and the centre of the input pupil $T$ is related to the position vector $O O_{i}$ of the sub pupil $i$ in the output pupil by

$T T_{i}=G \cdot O O_{i}$

where $O$ is the output pupil origin.

The $G$ factor gives the angular magnification of the instrument. In order to concentrate the energy around the focal point of the image plan, each beam diameter is expanded up to the limit of an overlapping between the different sub pupils in the output pupil plan. Figure 2 illustrates this configuration. When observing the beam $i$, the parameter $T T_{i}$ describing the telescope position in the input pupil plane is converted into the obliquity of the limited plane wave illuminating the image plane. Assuming 


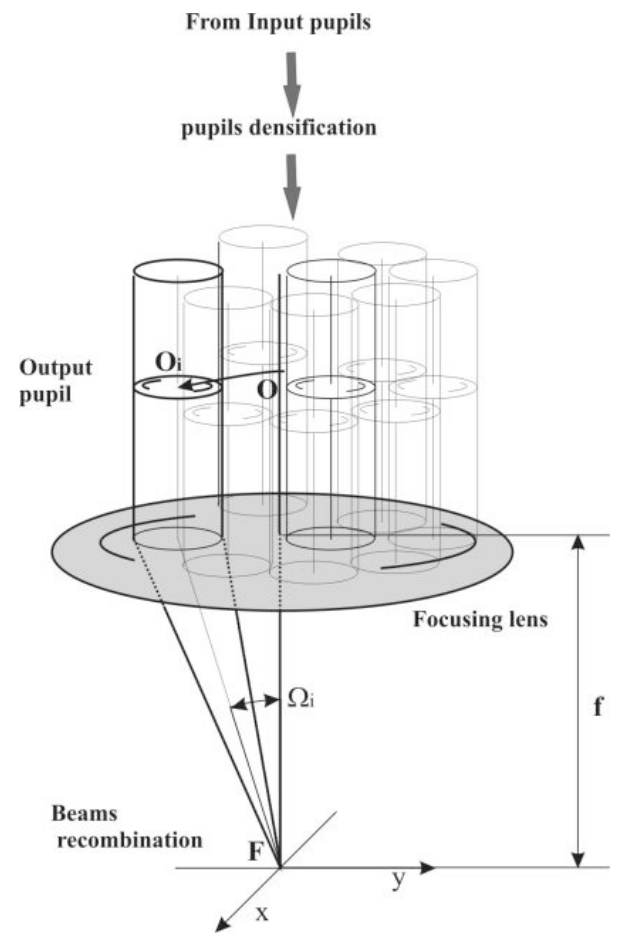

Fig. 2. Geometry of the densified pupil. Each sub pupil gives rise to a limited plane wave reaching the focal plane with a $\Omega_{i}$ obliquity.

that the paraxial conditions is applied, the corresponding twodimensional angle $i$ is equal to

$\Omega_{i}=\frac{O O_{i}}{f}$

where $f$ is the focal length of the focusing lens. In the image plane, the related limited plane wave can be expressed as

$E_{i}=a_{i} \cdot \exp \left(j \psi_{i}\right) \cdot d(X) \cdot \exp \left(j K_{i} \cdot X\right)$

with

$K_{i}=\frac{2 \pi}{\lambda} \frac{O O_{i}}{f}=\frac{2 \pi}{\lambda} \frac{T T_{i}}{G f}$

where $a_{i}$ and $\Psi_{i}$ are the modulus and input phase of beam $i, X$ is the position vector in the image plane with two coordinates $x$ and $y(X=(x ; y))$. In Labeyrie's configuration $d(X)$ denotes the spot field envelop resulting from the diffraction of one sub pupil (see Fig. 3) and is supposed to be identical for all beams. The last term $\exp \left(j K_{i} \cdot X\right)$ shows the linear variation of the phase in the image plan according to $\alpha_{i}$ and $\beta_{i}$ slope coefficients.

$K_{i}=2 \pi\left(\alpha_{i}, \beta_{i}\right)$

with

$\alpha_{i}=\frac{\left(O O_{i}\right)_{x}}{\lambda f} \quad$ and $\quad \beta_{i}=\frac{\left(O O_{i}\right)_{y}}{\lambda f}$

where $\left(O O_{i}\right)_{x}$ denotes the projection of vector along $x$ axis.

In this way, each position $T T_{i}$ of telescope drives the slope $\left(\alpha_{i}, \beta_{i}\right)$ of the corresponding wave front reaching the image plane. The phase evolution $\varphi_{i}(x, y)=K_{i} X$ can be analysed along different axes parallel to the $x$ axis. The phase variation $\varphi_{i}(x)$ is linear with an offset depending on the $y$ coordinate:

$\varphi_{i}(x)=2 \pi \cdot\left(\alpha_{i} \cdot x+\beta_{i} \cdot y\right)=2 \pi \cdot \alpha_{i} \cdot x+\varphi_{i 0}(y)$

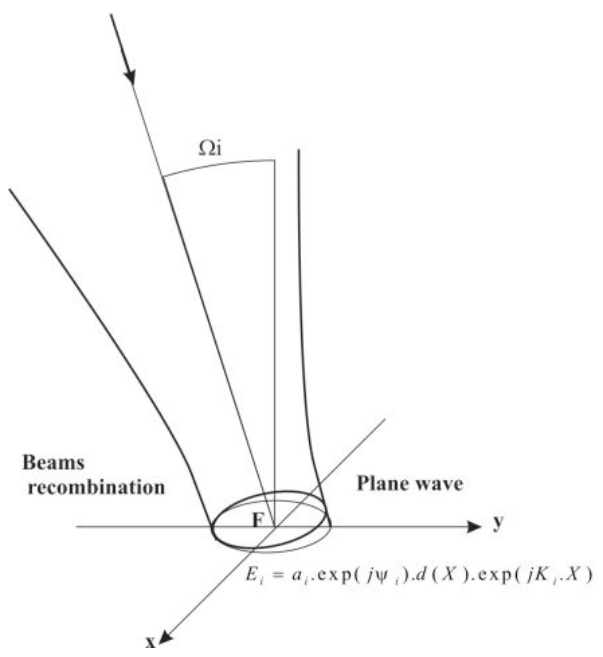

Fig. 3. Contribution of beam $i$ in the image plane. The optical field has a limited-plane wave structure with a diffraction envelope $d(X)$.

with

$\varphi_{i 0}(y)=\varphi_{i 0}(0 ; y)=2 \pi \cdot \beta_{i} \cdot y$.

As demonstrated by Labeyrie, the possibility of retrieving the image results from the coherent superposition of these different limited-plane waves and the convolution of the resulting point spread function (PSF) with the object's intensity distribution. The PSF is given by the intensity distribution corresponding to the coherent superposition of field $E_{i}$ in the image plane when the input pupil is illuminated by a plane wave with a wave vector perpendicular to the input pupil plane. In such conditions, the $a_{i}$ coefficients are identical (set to one, for example) and the $\Psi_{i}$ phase terms in Eq. (3) are equal to 0:

$$
\begin{gathered}
P S F(X)=\left|\sum_{i} E_{i}\right|^{2}=\left|\sum_{i} d(X) \cdot \exp \left(j K_{i} \cdot X\right)\right|^{2} \\
=|d(X)|^{2} \cdot\left|\sum_{i} \exp \left(j K_{i} \cdot X\right)\right|^{2} .
\end{gathered}
$$

If in the Labeyrie's configuration, $d(X)$ corresponds to the subpupil's far field, in the IRAN proposal this term is replaced by the sub-pupil function itself. In the both cases, this envelope term is responsible for the phase modulation range in the recombining plane. This is directly related to the optics dimension and will not be easy to adjust in a real experiment.

For a tilted point-like source illuminating the telescope array with a $\theta=\left(\theta_{x}, \theta_{y}\right)$ obliquity, the amplitudes $a_{i}$ remain constant, but the phase $\psi_{i}$ becomes

$\psi_{i}=\frac{2 \pi}{\lambda} T T_{i} \cdot \theta$

The total phase of the $E_{i}$ field can be written in the image plane as

$\psi_{i}+\varphi_{i}=\frac{2 \pi}{\lambda} T T_{i} \cdot \theta+\frac{2 \pi}{\lambda} \frac{T T_{i}}{G f} \cdot X$

This additional $\psi_{i}$ term induces a shift of the corresponding intensity distribution:

$I_{\theta}(X)=P S F(X+G f \theta)$ 

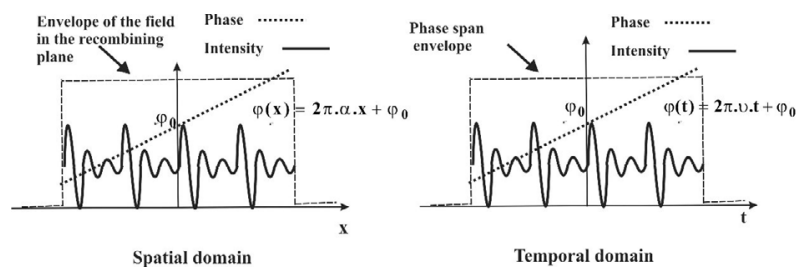

Fig. 4. Analogy between spatial and temporal interferences. In the first case (left) the fringes are displayed as a function of the position and the interferometer induces a spatial phase variation. In the second case (right), a temporal optical path modulation allows an observation as a function of time $t$. The frame in which fringes are observed results from a beam spatial dimension (left) and a limited optical path stroke (right).

For an extended object, the incoherent superposition of the different contribution of the object $O(\theta)$ leads to the image

$I(X) \propto \int O(\theta) \cdot P S F(X+G f \theta) \mathrm{d} \theta$.

Denoting $X_{0}=f \theta$, the image appears as a pseudoconvolution of the object intensity distribution with the PSF:

$I(X) \propto \int O\left(\frac{X_{0}}{f}\right) \cdot P S F\left(X+G X_{0}\right) \mathrm{d} X_{0}$.

Any process able to achieve such a recombination will provide an image with the same basic properties. The next paragraph demonstrates the possibility to obtain this result in the time domain. Note that the transposition in the time domain of the IRAN proposal could be achieved as well as in the Labeyrie's configuration with minor differences.

\section{From spatial to temporal domain}

The aim of this paragraph is to demonstrate the equivalence of the temporal and spatial approaches. According to the design of the experimental set up, an interferometric signal can be displayed as a function of time or space depending on whether the interferometric mixing has been achieved in a multiaxial or coaxial configuration. This point is illustrated in Fig. 4 for a twodimensional representation. The two techniques are fully equivalent as long as the numbers of spatial or temporal samples are identical and the full integration times are the same for the two experimental configurations.

For hypertelescopes in the spatial case, the linear phase modulations $\varphi_{i}(x)$ result from a variable optical path that is different related to the $\Omega_{i}$ tilts of the optical fields $E_{i}$ reaching the observation plane and coming from beam $i$.

In the temporal case, the phase modulation $\varphi_{i}(t)$ can be generated thanks to an optical-path variation induced by optical-path modulators that are linearly actuated as a function of time as shown in Fig. 5:

$\varphi_{i}(t)=2 \pi \cdot v_{i} \cdot t+\varphi_{i 0}(y)$.

Using a temporal frequency $v_{i}$ proportional to the $\alpha_{i}$ slope allows us to propose a temporal configuration equivalent to the one in the spatial domain. For this purpose, the frequency $v_{i}$ of the phase modulation has to be selected as proportional to the $\alpha_{i}$ coefficient with an $A=\frac{v_{i}}{\alpha_{i}}$ proportional factor:

$v_{i}=A \frac{\left(O O_{i}\right)_{x}}{\lambda \cdot f}=A \frac{\left(T T_{i}\right)_{x}}{\lambda \cdot f \cdot G}$
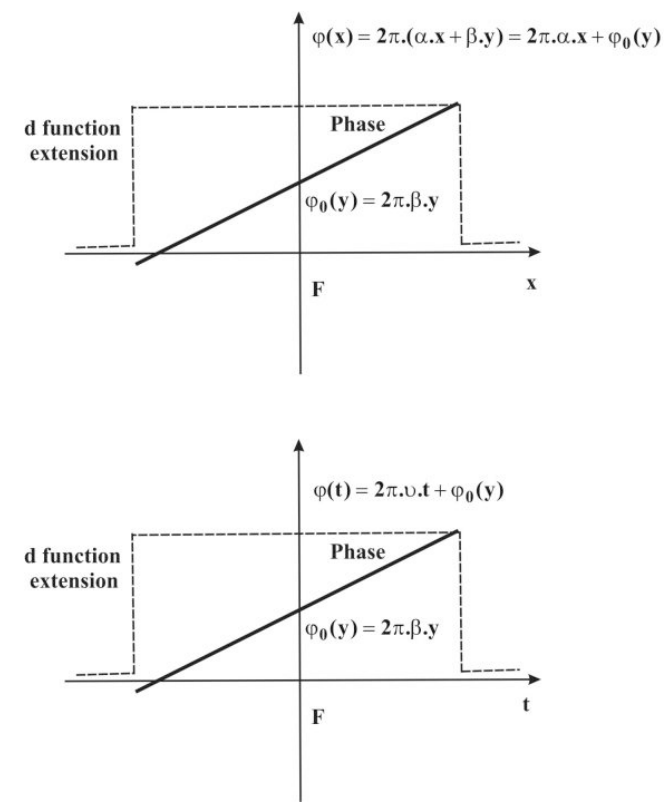

Fig. 5. Phase variation of one beam for spatial and temporal modulations. Using proportional slopes between the two configurations allows us to display similar interferences patterns with only a scale ratio between the two horizontal axes. The phase span is driven by the diffraction effect and the optical path stroke.

Following this spatial to temporal transposition the optical field

$E_{i}=a_{i} \cdot \exp \left(j \psi_{i}\right) \cdot d(X) \cdot \exp \left\{j\left[2 \pi \cdot \alpha_{i} \cdot x+\varphi_{i 0}(y)\right]\right\}$

is replaced by

$E_{i}=a_{i} \cdot \exp \left(j \psi_{i}\right) \cdot d(t) \cdot \exp \left\{j\left[2 \pi \cdot v_{i} \cdot t+\varphi_{i 0}(y)\right]\right\}$,

where the $d(t)$ function allows us to limit the phase variation as needed. The coherent superposition of all the beams can be analyzed along $x$ axis. The $A$ factor gives the relationship between the spatial parameter $x$ and time $t$

$x=A \cdot t$

with

$\varphi_{i}(t)=2 \pi \cdot v_{i} \cdot t+\varphi_{i 0}(y)=\frac{2 \pi}{\lambda} \frac{T T_{i}}{G f} \cdot(A t ; y)$.

As in the spatial domain, the image is obtained by using the PSF given by

$P S F(t ; y)=\left|\sum_{i} E_{i}\right|^{2}=\left|\sum_{i} d(t) \cdot \exp \left\{j\left[2 \pi \cdot v_{i} \cdot t+\varphi_{i 0}(y)\right]\right\}\right|^{2}$.

For a tilted point like source with a $\theta=\left(\theta_{x} ; \theta_{y}\right)$ obliquity, the total phase of the $E_{i}$ field becomes

$\psi_{i}+\varphi_{i}=\frac{2 \pi}{\lambda} T T_{i} \cdot \theta+\frac{2 \pi}{\lambda} \frac{T T_{i}}{G f} \cdot(A t ; y)$

As previously, the corresponding intensity distribution is a shifted PSF:

$I_{\theta}(A t ; y)=P S F[(A t ; y)+G f \theta]$ 


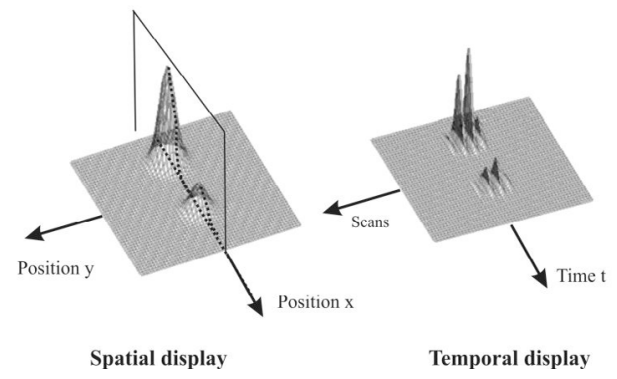

Fig. 6. Temporal display versus spatial display. The two-dimensional spatial display can be replaced by a temporal one. As long as the time is a one-dimensional parameter, a set of scans is necessary to retrieve a time-multiplexed image like in a video monitor.

The incoherent superposition of the different contribution of the object $O$ leads to the image with

$$
\begin{array}{r}
I(A t ; y) \propto \int O(\theta) \cdot \operatorname{PSF}[(A t ; y)+G f \theta] \mathrm{d} \theta \\
\propto \int O\left(\frac{X_{0}}{f}\right) \cdot P S F\left[(A t ; y)+G X_{0}\right] \mathrm{d} X_{0}
\end{array}
$$

where $X_{0}=f \theta$.

As previously mentioned, the image appears as a pseudoconvolution of the object's intensity distribution with the PSF, but now this one is displayed as a function of time. This result demonstrating the full equivalence between spatial and temporal display is illustrated in Fig. 6.

To show a two-dimensional image, the $\varphi_{i 0}(y)$ phase is modified step by step so as to successively display the different sections of the image using a set of scans. The information provided along the $x$ axis at a given position $y$ is fully equivalent to the one observed as a function of time $t$ for the scan related to the same $\varphi_{i 0}(y)$ value. The image is temporally multiplexed as for a video monitor. In the spatial domain, the $x$ span is determined by the diffraction field distribution related to the sub-pupil geometry. In the temporal domain, the field of investigation is determined by the phase-modulation extension directly driven by the span of the optical-path modulator. It can be adjusted very easily and reduced up to 0 opposite to the spatial configuration where the limitation results from the beam dimensions.

\section{Implementing a time-modulated instrument}

Consequently the time-modulated version of a hypertelescope can be drawn as proposed in Fig. 7. This sketch uses optical fibers to propagate the light, but classical processes using mirror trains, air delay lines, and classical beam splitters are just as convenient. The light is picked up at the $\mathrm{N}$ telescope foci and passes through optical-path modulators before being recombined using an $\mathrm{N}$ to 1 beam combining coupler in a coaxial configuration. The optical-path modulations are generated using optical fiber stretchers inducing an optical-path variation in order to generate the convenient phase modulations as previously mentioned:

$\delta_{i}=\frac{A\left(O O_{i}\right)_{x}}{f} \cdot t+\frac{\lambda}{2 \pi} \cdot \varphi_{i 0}(y)=\frac{A\left(O O_{i}\right)_{x}}{f} \cdot t+\frac{\left(O O_{i}\right)_{y} \cdot y}{f}$.

Servo-control systems have been developed for these kinds of applications (Reynaud et al. 1992 and Delage et al. 2000) and can monitor the linearity of the optical-path variation as a function of time with nanometric accuracy. For each scan, the offset $\frac{\left(O O_{i}\right)_{y} \cdot y}{f}$ is modified in order to display the signal that would be spatially observed for the corresponding $y$ position.

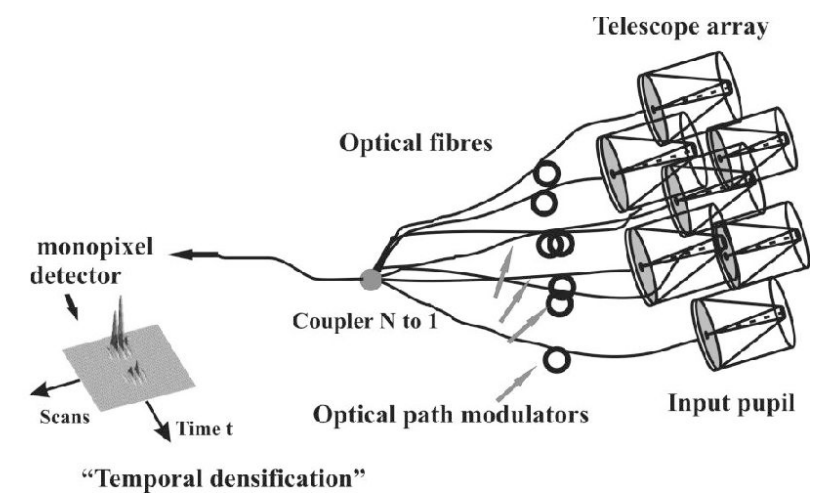

Fig. 7. Proposal for a temporal hypertelescope using optical fibers and a guided coupler acting as a combiner. The optical-path modulators induce the phase variations needed to form an image. The image is temporally multiplexed.

\section{Versatility of a temporal display}

For a ground mission, the classical hypertelescope technique needs to continuously reconfigure the output apertures due to the earth rotation. The telescopes map, as seen from the scientific target, is continuously modified, making the output pupil reconfiguration necessary as long as hypertelescopes use a homothetic reconfiguration of the sub-pupil centres. For a space mission the reconfiguration of the input pupils map is of interest and makes a continuous output pupil reconfiguration necessary. This hardware operation is quite complex and uses a lot of time. Conversely, in the time domain, the change in the input pupil configuration is followed by a simple change in optical-path modulation slopes and offsets. This operation can be achieved using the software that monitors the optical-path servo-control systems. For a space mission, the opportunity to change the input pupil configuration can be easily investigated thanks to this experimental functionality. In this way, it is possible to take advantage of this versatility to use the redundant or non redundant configurations according to the (faint; extended) or (bright; sharp) science targets (see Lardiere et al. 2007).

\section{From the hypertelescope to a nulling interferometer}

The second advantage deals with the easy control of the opticalpath span related to a high-densification potential. In the spatial domain, the phase span is related to the slope of the wave front and diffraction spot diameter over which the beam is spread. Since the spatial densification process is limited by the overlapping of sub-apertures in the output plan, the span limit results from the diffraction pattern of each sub pupil. Conversely, in the time domain, the full variation of the phase can be reduced as much as necessary by reducing the span of the optical path modulation. In this way, it is possible to focus the investigation on a limited part of the field. This point is illustrated in Fig. 8. The servo-control system is adjusted in order to limit the scan, this operation being achieved by software. This functionality can be investigated up to a zero optical-path modulation. In such configuration, the instrument is pointing only one point $(x, y)$ of the image (see right part of Fig. 8) setting the optical paths equal to

$\delta_{i}=\frac{\left(O O_{i}\right)_{x}}{f} \cdot x+\frac{\left(O O_{i}\right)_{y}}{f} \cdot y$.

In this case, the instrument is operating as a nulling interferometer (Bracewell 1978). The light coming from a planet can be 

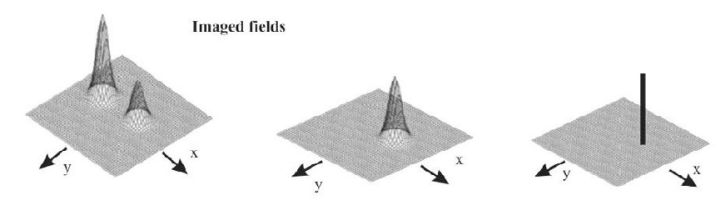

Temporal signals ( One scan

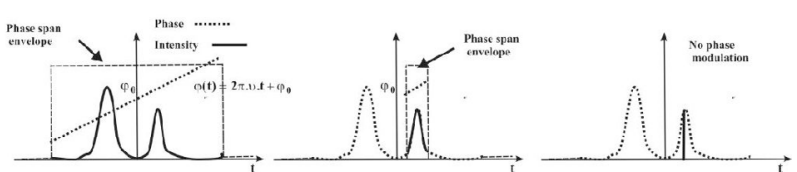

Fig. 8. The optical path stroke on each modulator can be adjusted in order to modify the field over which the image is displayed (from left to right). Reducing these modulations allows concentration of this imaging process on a limited area (centre). Setting the different phases to the proper values (left) enables us to operate in a nulling configuration thereby analysing only one point of the scene to be observed.

selected in the $(x, y)$ point of the field, while the light of its parent star is canceled.

\section{Perspectives and conclusion}

As presented in this paper, the temporal hypertelescope has not been optimized for sensitivity. This presentation has focused on the principle and the related advantages of the temporal modulation and densification using a simple $\mathrm{N}$ to 1 combiner for pedagogical reasons. For a future instrument, the $\mathrm{N}$ to 1 combiner is not efficient as long as the N-1 missing outputs lead to a large loss of energy. At the present time, an $\mathrm{N}$ to $\mathrm{N}$ combiner is being studied in our lab. Two options are being investigated using field or amplitude division interferometers. The technological possibilities are numerous and will need to be evaluated. The main goal of this ongoing study is to propose a beam combiner that will give rise to a set of outputs with a global preservation of the energy. In this way, the temporal hypertelescope will reach the same sensitivity as a spatial one.

The second point to be investigated is the optimization of each output response. In the frame of a collaboration with a mathematics department in our institute, we have begun a study to improve the PSF rejection ratio using optimization processes for the redundant versus non-redundant input pupil geometries.

Finally, the internal metrology has to be designed the specific requirements of such an instrument. A typical optical path accuracy in the range of $\lambda / 100-\lambda / 1000$ will be necessary. A servo-control system based on the simultaneous propagation of a metrology light and the science one could be used. This problem is common with all the phased arrays devoted to high resolutionimaging.

Before the finalization of these three studies, the new temporal configuration of a hypertelescope needs to be experimentally tested in order to valid the related potential improvements. A breadboard is under construction for a laboratory test. The possibility of reconfiguring the pupil is a very attractive functionality that will be investigated. This functionality both answers the problem of the continuous remapping of a telescope array for a ground-based instrument and proposes a very high flexibility for the reconfiguration of a space instrument. The next feature to be investigated will be conversion of the temporal hypertelescope into a nulling interferometer by freezing the phase modulation at the position that corresponds to a science target.

Acknowledgements. This work is supported by the Centre National d'Étude Spatiale (CNES).

\section{References}

Aristidi, E., Vakili, F., Abe, L., et al. 2004, SPIE 5491, New Frontiers in Stellar Interferometry, Ed. W.A. Traub, Bellingham, SPIE, 763

Bracewell, R. N. 1978, Nature, 274, 780

Delage, L., Reynaud, F., \& Lannes, A. 2000, Appl. Opt., 39, 6406

Gillet, S., Riaud, P., Lardière, O., et al. 2003, A\&A, 400, 393

Labeyrie, A. 1996, A\&AS, 118, 517

Lardiere, O., Martinache, F., \& Patru, F., 2007, MNRAS, in press

Pedretti, E., Labeyrie, A., Arnold, L., et al. 2000, A\&AS, 147, 285

Reynaud, F., Alleman, J. J., \& Connes, P. 1992, Appl. Opt., 31, 19, 3736

Traub, W. A. 1986, Appl. Opt. 25, 528

Vakili, F., Aristidi, E., Abe, L., \& Lopez, B. 2004, A\&A, 421, 147 